\title{
Idiopathic Ovarian Torsion in a Young Female
}

\author{
Raj Lakshmi Nalam, Shiva Kumar KS, Balakrishna BV
}

Sri Satya Sai Institute of Higher Medical Sciences, Prasanthigram, Puttaparthi-515134, Andhra Pradesh, India.

\section{Corresponding Author:}

Dr. Raj Lakshmi Nalam

Email: rajlakshmi.nalam@gmail.com

This is an Open Access article distributed under the terms of the Creative Commons Attribution License (creativecommons.org/ licenses/by/3.0).

Received

Accepted

Published

April 2, 2019

October 5, 2019

November 10, 2019

\begin{abstract}
Background: Ovarian torsion is a surgical emergency. It remains one of the most challenging diagnosis in an emergency room. Prompt diagnosis and surgical intervention are key to good outcomes. Case Report: We report a case of 32 year old unmarried woman who presented to the emergency room with pain in right iliac fossa and vomiting. Prompt diagnosis with help of ultrasonography and surgical intervention in form of laparoscopic detorsion and oophoropexy helped in salvaging the adnexa. Conclusion: Idiopathic ovarian torsion at this age is rare and has catastrophic effect on future fertility. Ultrasonography is the primary imaging modality. We also want to highlight the importance of prompt surgical intervention in form of detorsion with oophoropexy to prevent subsequent torsion.
\end{abstract}

Keywords: Adnexa Uteri, Ovarian Diseases, Fertility, Laparoscopy, Pain.

\section{Introduction}

Adnexal torsion includes torsion of the ovary, tube or both, with resulting arterial, venous, or lymphatic obstruction. Ovarian torsion is the fifth most common gynaecological emergency with a reported prevalence of $2.7 \%$ in all cases of acute abdominal pain [1]. Prompt diagnosis and surgery may prevent irreversible adnexal damage. The importance of this case report lies in the rarity of ovarian torsion at this age; isolated ovarian torsion are common in pediatric age group. Ultrasonography is the first line imaging modality for patients with suspected ovarian torsion. Laparoscopic detorsion alone is not sufficient as the anatomical factors predisposing to torsion still persist, hence oophoropexy should be considered to prevent future torsion of normal ovary.

\section{Case Report}

A 32 years old unmarried woman presented to the emergency department in the morning on $22^{\text {nd }}$ day of her menstrual cycle with severe pain in right iliac fossa since the day before night. The pain was severe and constant without any radiation and was associated with three episodes of vomiting. Patient did not have any associated genitourinary or gastrointestinal symptoms. She gave history of physical exertion continuously for one week before this episode of abdomen pain. She did not have any similar episodes in the past.

On examination, she was afebrile, pulse rate was 96/min, blood pressure of 130/80 $\mathrm{mm} \mathrm{Hg}$. Her abdomen was soft, but had tenderness in right iliac fossa, there was no guarding, rigidity, or fluid thrill. Bowel sounds were present. Her total leucocyte counts were $11,200 / \mathrm{mm}^{3}$, urine microscopy was normal. Urine pregnancy test was negative. ESR was $80 \mathrm{~mm} \mathrm{Hg}$. Ultrasound abdomen and pelvis showed enlarged right ovary with compromised venous flow [Fig.1]. Probable diagnosis of right sided ovarian torsion was made.

With probable diagnosis of right ovarian torsion, patient was posted for diagnostic laparoscopy. Intra-operative right adnexa was twisted twice over it's pedicle [Fig.2]. Ovary 
was healthy without any cyst or any features of necrosis. Right fallopian tube was edematous. Detorsion was done and the right ovary was fixed to the ipsilateral round ligament [Fig.3]. The other ovary and fallopian tube were normal. Patient had a complete post-operative recovery. Skin sutures were removed on $7^{\text {th }}$ post-operative day.

\section{Discussion}

Adnexal torsion remains one of the most challenging diagnosis in the emergency room and is based on clinical symptoms, examination findings and imaging modalities. In 2005, White $\mathrm{M}$ et al. [2] conducted a retrospective study over a period of ten years with fifty two cases of ovarian torsion and found that the main clinical features at presentation were sudden onset of pain in $87 \%$ of the cases, nausea and vomiting in $59 \%$ of the cases and palpable abdominal mass in $62.2 \%$ of the cases. In this case, abdominal pain and vomiting were the presenting symptoms, but these symptoms are seen in several other conditions like appendicitis, cholecystitis, PID, urinary collecting system calculi, and ruptured benign adnexal cysts. Hence, high degree of clinical suspicion is required to make the diagnosis.

The mechanism of torsion of normal ovaries in the absence of cysts or masses is unclear. This has been found in patients of all ages, particularly in pre-menarchal girls. The utero-ovarian ligament is normally elongated in pre-menarchal girls and then shortens as they mature through puberty. Hypermobility due to an elongated utero-ovarian ligament and hyper-laxity of mesosalpinx or mesoovarium may be contributing factors [3]. Right side ovarian torsion is common ( $70 \%$ of the cases), this may be explained by the fact that the right utero-ovarian ligament is physiologically longer than the left, or by the presence of the sigmoid colon on the left which reduces the space needed for torsion to occur. Initially venous and lymphatic occlusion occurs and consequently the muscular arteries get occluded as a resultant of progressing edema.

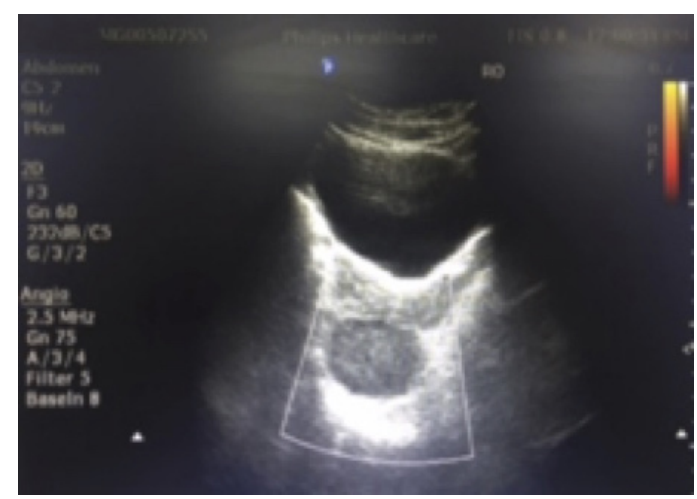

Fig.1: Ultrasound showed enlarged right ovary with compromised venous flow.

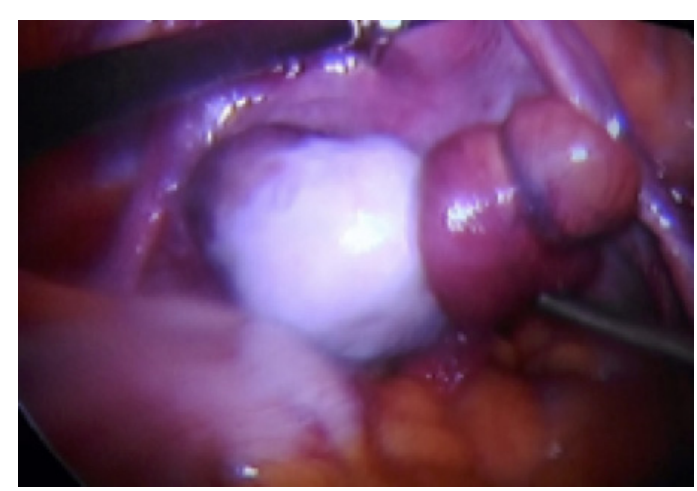

Fig.2: Intra-operative right adnexa twisted twice over it's pedicle.

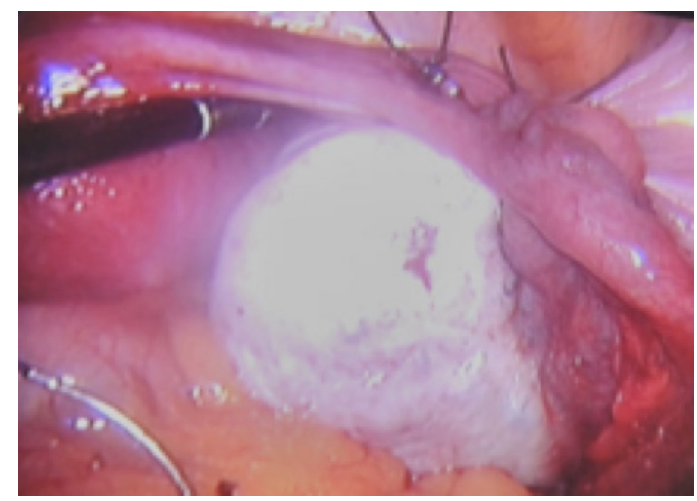

Fig.3: Right ovary fixed to the ipsilateral round ligament after detorsion.

The same mechanism explains the torsion in this case as ovary was normal without any cysts. These anatomical factors resulting in torsion after so many years after menarche makes this case unique. 
The diagnosis of ovarian torsion is challenging as the clinical parameters yield low sensitivity and specificity. Pelvic ultrasound is the first-line imaging modality for patients with suspected ovarian torsion. Ultrasound features are frequently described as unilateral ovarian enlargement and edema, with peripherally arranged follicles, complete absence of perfusion may be a relatively late event. Abnormal doppler flow is highly suggestive of torsion, but normal flow on Doppler does not exclude an adnexal torsion [4]. Hence, the presence of blood flow within the ovary does not exclude the diagnosis of torsion [5]. Doppler flow studies should not delay surgical exploration in the setting of suggestive signs and symptoms. Pelvic magnetic resonance imaging (MRI) or computed tomography (CT) scan is not usually ordered for evaluation for adnexal torsion.

The goals of the intra-operative evaluation are to confirm the presence of torsion and evaluate the viability of the ovary and tube. Since most of the cases are seen in women of reproductive age group, surgeons should always try to salvage the adnexa. Conservative treatment consists of detorsion of the adnexa, followed by treatment of the etiology like puncturing of a cyst or intra-peritoneal cystectomy [6]. Oophorectomy should be reserved for necrotic/ gelatinous/dead tissue.

Unlike orchidopexy for testicular torsion, whether or not to perform oophoropexy following de-torsion of normal adnexae is controversial. In cases where recurrent torsion has occurred, oophoropexy has been shown to be effective in reducing the recurrence rate [7]. Oophoropexy involves fixing of ovary to ligamentous structures like round ligament, ovarian ligament or uterosacral ligament. Current adnexa sparing laparoscopic management of adnexal torsion by simply untwisting may predispose to recurrent torsion in case of normal adnexa [8]. Hence, in this patient we chose to perform oophoropexy. In patients with congenitally long utero-ovarian ligament which is commonly seen in pediatric population and those patients with idiopathic ovarian torsion, oophoropexy can be used to prevent recurrent torsion.

\section{Conclusion}

Diagnosis of adnexal torsion requires high degree of clinical suspicion. Ultrasound with Doppler is helpful in its diagnosis. Laparoscopy is the gold standard for evaluation and management of adnexal torsion. Conservative management including detorsion with or without cystectomy is the preferred treatment. A complete resection of tube/ovary/both is performed when the tissue is gangrenous or when malignancy is suspected or if woman has completed her family. Early diagnosis and timely management of adnexal torsion should be done to preserve the reproductive function. In patients with idiopathic ovarian torsion, oophoropexy should be considered as it prevents recurrent torsion.

Contributors: RLN: manuscript writing, and patient management; SKKS: reviewing the literature and operative surgeon; BBV: critical inputs into the manuscript and radiological diagnosis. RLN will act as a study guarantor. All authors approved the final version of this manuscript.

Funding: None; Competing interests: None stated.

\section{References}

1. Mishra VV, Nanda S, Nawal R, Choudhary S. Unusual presentation of twisted ovarian cyst. J Midlife Health. 2016;7(1):31-33.

2. White M, Stella J. Ovarian torsion: 10-year perspective. Emerg Med Australas. 2005;17(3):231-237.

3. Davis AJ, Feins NR. Subsequent asynchronous torsion of normal adnexa in children. J Pediatr Surg. 1990;25(6):687-689.

4. Pena JE, Ufberg D, Cooney N, Denis AL. Usefulness of Doppler sonography in the diagnosis of ovarian torsion. Fertil Steril. 2000;73(5):1047-1050.

5. Shadinger LL, Andreotti RF, Kurian RL. Preoperative sonographic and clinical characteristics as predictors of ovarian torsion. J Ultrasound Med. 2008;27(1):7-13.

6. Gocmen A, Karaca M, Sari A. Conservative laparoscopic approach to adnexal torsion. Arch Gynecol Obstet. 2008;277(6):535-538. 
7. Fuchs N, Smorgick N, Tovbin Y, Ben Ami I, Maymon R, Halperin R, Pansky M. Oophoropexy to prevent adnexal torsion: how, when and for whom? J Minim Invasive Gynecol. 2010;17(2):205-208.
8. Pansky M, Smorgick N, Herman A, Schneider D, Halperin R. Torsion of normal adnexa in postmenarchal women and risk of recurrence. Obstet Gynecol. 2007;109(2 Pt 1):355-359. 